\section{$\mathbf{m} / \mathbf{s}$}

médecine/sciences $1997 ; 13: 1393-5$

\title{
L'HYPERCHOLESTÉROLÉMIE ET LE CLINICIEN
}

\section{Gérard Turpin}

\section{RÉFÉRENCES}

1. Brown MS, Goldstein JL. Familial hypercholesterolemia : defective binding of lipoproteins to cultured fibroblasts associated with impaired regulation of 3-hydroxy-3-methyl glutaryl co-enzyme A reductase activity. Proc Natl Acad Sci USA 1974 ; 71 : 788-92.

2. Kahn A. L'hypercholestérolémie familiale, de la maladie au gène. Med Sci $1985 ; 1$ : 388 .

3. Innenarity TL, Mahley RW, Weisoraber KH, et al. Familial defective apolipoprotein B 100 : a mutation of apolipoprotein B that causes hypercholesterolemia. J Lipid Res 1990 ; 31 : 1337-49.

4. Benlian P. Génétique des dyslipidémies. Approche gène-candidat, vol. 1. Paris : Éditions Inserm, 1996 : $253 \mathrm{p}$.

5. Turpin G. Pourquoi, quand, comment traiter les dyslipoprotéinémies ? Vol. 1. Dijon : Impressions, 1997: $260 \mathrm{p}$.

6. Tybjaerg-Hansen A, Humphries SE. Familial defective apolipoprotein B 100 : a single mutation that causes hypercholesterolemia and premature coronary artery disease. Atherosclerosis 1992; 96 : 91-107.

7. Stalenhoef AFH, Defesche JC, Kleinveld HA, Demacker PNM, Kastelein JJP. Decreased resistance against in vitro oxidation of LDL from patients with familial defective apolipoprotein B 100. Arterioscler Thromb $1994 ; 14$ : 489-93.

8. Varret M, Rabès JP, Boileau C. L'hypercholestérolémie familiale 25 ans. I. Défauts du récepteur des LDL. Med Sci 1997 ; 13 : 1399-408.

9. Brown MS, Goldstein JL. A receptor-mediated pathway for cholesterol homeostasis. Science 1986 ; 232 : 34-47.

10 . Valère T. Thérapie génique : le point sur les essais cliniques. Med Sci 1996; 12 : 73-83.

\section{ADRESSE ET TIRÉS À PART}

G. Turpin : professeur des universités-praticien hospitalier, chef du service d'endocrinologie-métabolisme. Groupe hospitalier Pitié-Salpêtrière, 45-83, boulevard de l'Hôpital, 75651 Paris Cedex 13, France. es médecins cliniciens ont toujours une grande admiration pour les découvertes fondamentales qui font progresser d'un bond les connaissances et la compréhension de certaines maladies. A plus ou moins long terme, cela débouche sur une meilleure prise en charge clinique et thérapeutique des patients. Trop souvent encore, la médecine est considérée comme une science point trop exacte... Il n'en est rien pour certaines maladies métaboliques, et parmi celles-ci, l'hypercholestérolémie familiale. Les remarquables travaux de Brown et Goldstein (Dallas, TX, USA), commencés dès les années 1970 [1], ont non seulement permis de comprendre de façon parfaite la pathogénie de certaines hypercholestérolémies familiales (type IIa), mais ont également conduit à la mise au point de molécules thérapeutiques remarquablement efficaces dans cette affection (les statines) et permettront peut-être de déboucher dans un avenir plus ou moins proche sur une thérapie génique dès que des problèmes techniques encore importants seront maîtrisés. La communauté scientifique ne s'est pas trompée sur l'importance de cette découverte puisqu'elle a décerné le prix Nobel en 1985 à ces deux auteurs [2]. Des études ultérieures ont précisé que les hypercholestérolémies n'étaient pas toutes liées à une anomalie du gène du récepteur LDL. C'est le mérite de Innenarity et al. (San Francisco, CA, USA) d'avoir entrevu les anomalies possibles du gène de l'apolipoprotéine B [3], en sachant que ces deux situations où la physiopathologie est bien démontrée ne rendent pas compte de tous les problèmes observés en clinique.

\section{Pouvoir athérogène des LDL}

Les LDL (low density lipoproteins) se situent au bout de la chaîne catabolique des VLDL (very low density lipoproteins). Leurs caractères physico-chimiques sont parfaitement connus [4, 5] et de nombreux arguments permettent d'affirmer que les LDL sont des lipoprotéines hautement athérogènes $[5]$.

\section{- Des preuves expérimentales}

D'abord : l'augmentation de la cholestérolémie totale, par augmentation de la fraction LDL-cholestérol, induite par un régime riche en cholestérol et en graisses saturées, entrâ̂ne chez l'animal d'expérience une athérosclérose accélérée et prématurée.

\section{- Des preuves épidémiologiques}

Toutes les études épidémiologiques mettent en évidence une corrélation positive, statistiquement significative, entre la quantité de cholestérol et de graisses saturées dans l'alimentation et les concentrations de cholestérol total et de LDL-cholestérol plasmatiques ; une corrélation positive, statistiquement significative, entre la quantité de cholestérol et de graisses saturées dans l'alimentation et la fréquence des complications cardiovasculaires ischémiques, coronariennes en particulier ; et, enfin, une corrélation positive, statistiquement significative, entre les concentrations de cholestérol total et de LDL-cholestérol plasmatiques et la fréquence des complications coronariennes $(\mathrm{m} / \mathrm{s}$ $n^{\circ} 2$, vol. 11, p. 293).

- Des preuves cliniques et biologiques Les hyperlipoprotéinémies (l'hypercholestérolémie pure de type IIa et 
l'hyperlipidémie combinée ou mixte de type IIb) comportant une augmentation (isolée ou non) du cholestérol total par augmentation du LDL cholestérol sont fortement athérogènes. Dans l'hypercholestérolémie pure de type IIa, il existe le plus souvent une corrélation positive et statistiquement significative entre l'augmentation du LDL cholestérol et la fréquence et la gravité des complications cardiovasculaires ischémiques.

\section{- Des preuves apportées par les études de prévention}

Qu'il s'agisse des études de prévention primaire ou de prévention secondaire, la diminution du cholestérol total par diminution du LDL cholestérol induite par le régime et/ou des médicaments entrâ̂ne une baisse de la morbidité coronarienne. Les études récentes effectuées avec les statines, en prévention primaire ou secondaire ont confirmé que la diminution significative du LDL cholestérol entraînait non seulement une baisse de la morbidité coronarienne, mais aussi de la mortalité coronarienne et de la mortalité totale $\left(\mathrm{m} / \mathrm{s} n^{\circ} 2\right.$, vol. 12 , p. 244).

- Enfin, les conclusions des études de régression de l'athérosclérose

De nombreuses études effectuées avec la cholestyramine, l'acide nicotinique, les statines et les dérivés des fibrates montrent que, au prix de la diminution significative du LDL cholestérol (associée ou non à une baisse des triglycérides et à une augmentation du HDL cholestérol), il est possible d'obtenir un ralentissement de la progression des lésions d'athérosclérose au niveau des artères coronaires, carotides, des membres inférieurs, ou une stabilisation, voire une véritable régression de ces lésions. Il n'y a pas toujours de parallélisme entre la diminution rapide, dès le sixième mois de l'étude, de la fréquence des complications coronariennes et la faible régression anatomique de la plaque. C'est le contenu lipidique de la plaque d'athérosclérose qui est surtout diminué, d'où une stabilisation de la plaque qui est dès lors moins

\section{Diagnostic des hypercholestérolémies}

Il est donc fondamental, pour le clinicien, d'affirmer l'augmentation du LDL-cholestérol. Or, dans ces périodes de restriction budgétaire, les Références Médicales Opposables (RMO) disent qu'il faut demander lors du premier examen biologique un simple dosage du cholestérol total (CT) et des triglycérides (TG). Ce n'est que s'il y a une anomalie de l'un et/ou de l'autre que l'on complétera par un contrôle plus complet comportant en outre le dosage du HDL cholestérol (C HDL) et de l'apolipoprotéine B. En fait, les dosages d'apo B et apo AI semblent moins informatifs que le dosage du HDL cholestérol. En pratique, on demandera donc la détermination au bout de 12 heures de jeûne de l'aspect du sérum à jeun, des concentrations de cholestérol total et de triglycérides, de la concentration de HDL cholestérol (par une technique de précipitation) et l'on calculera le LDL cholestérol (C LDL) par la formule classique $\mathrm{C}$ LDL $=\mathrm{CT}-\mathrm{C}$ HDL - TG/5 (g/l) (formule valable à condition que les TG soient inférieurs à $4 \mathrm{~g} / 1$ ).

Cela permettra de préciser dans un premier temps le type de l'hyperlipoprotéinémie ; deux hyperlipidémies s'accompagnent d'une augmentation des LDL : l'hypercholestérolémie pure (type IIa) et l'hyperlipidémie combinée ou mixte (type IIb).

Il faut savoir que le cholestérol total peut varier de 0,20 gramme/litre d'un jour à l'autre et qu'il est bon, sauf urgence, d'avoir deux ou trois contrôles biologiques sous régime libre avant de débuter le traitement. Cependant, dans l'hypercholestérolémie pure, les anomalies lipidiques plasmatiques sont très stables sous régime libre, avec pratiquement la même concentration de cholestérol total d'un bilan à l'autre. L'hypercholestérolémie pure ne s'accompagne pas en général d'anomalie du métabolisme des hydrates de carbone et des purines. La «formule » lipidique de l'hypercholestérolémie pure est très stéréotypée et est caractérisée, au bout de 12 heures de jeûne, par un sérum clair; une augmentation du cholestérol total, par augmentation de la fraction athérogène LDL cholestérol et, donc, de l'apolipoprotéine $\mathrm{B}$; des triglycérides normaux; un HDL cholestérol normal ou diminué selon les cas (l'apolipoprotéine $A_{I}$ étant modifiée de façon parallèle), et une augmentation exclusive des bêtalipoprotéines en électrophorèse sur gel d'agarose (type IIa de la classification internationale).

En revanche, on observe, dans l'hyperlipidémie combinée de type IIb, un sérum opalescent; une augmentation du cholestérol total, par augmentation de la fraction athérogène LDL cholestérol, et, donc de l'apolipoprotéine B ; une élévation à peu près parallèle des triglycérides; comme les triglycérides sont élevés, le HDL cholestérol est diminué, sauf dans trois conditions qui peuvent augmenter à la fois les triglycérides et le HDL cholestérol : l'alcoolisme chronique, l'hypothyroïdie, la prise orale d'œestrogènes; une augmentation des bêta et pré-bêta-lipoprotéines en électrophorèse sur gel d'agarose (type IIb de la classification internationale). Les LDL sont souvent petites et denses, c'est-à-dire plus oxydables et plus athérogènes.

\section{Traitement des hypercholestérolémies}

Une telle classification du désordre doit permettre de déboucher sur un traitement adapté, d'abord diététique: «anticholestérol » dans l'hypercholestérolémie pure (pauvre en cholestérol et en graisses saturées et enrichi en graisses mono et polyinsaturées), et également pauvre en hydrates de carbone à index glycémique élevé dans l'hyperlipidémie combinée ou mixte. Ce traitement diététique doit le plus souvent être complété par une thérapie médicamenteuse : statine et/ou résine échangeuse dans l'hypercholestérolémie pure, statine ou fibrate de deuxième génération dans l'hyperlipidémie combinée ou mixte.

Dans tous les cas, le suivi clinique et biologique régulier est nécessaire. Les niveaux d'intervention thérapeutique ont été récemment précisés en fonction de la concentration de LDL-cholestérol, car il serait illogique de trai- 
ter tous les patients de la même façon, sans tenir compte de l'existence d'autres facteurs de risque cardiovasculaire, d'antécédents cardiovasculaires familiaux, et de la situation cardiovasculaire primaire ou secondaire. En pratique, il est conseillé d'associer un hypolipidémiant si, malgré le régime : le LDL cholestérol reste supérieur à 2,20 g/l chez le sujet n'ayant pas d'autre facteur de risque, en prévention primaire ; le LDL cholestérol reste supérieur à $1,90 \mathrm{~g} / \mathrm{l}$ chez le sujet ayant un facteur de risque (le sexe masculin en est un), en situation de prévention primaire ; le LDL cholestérol reste supérieur à $1,60 \mathrm{~g} / 1 \mathrm{chez}$ le sujet ayant plusieurs facteurs de risque, en prévention primaire, et le LDL cholestérol reste supérieur à $1,30 \mathrm{~g} / 1$ dans les deux sexes si l'on est en situation de prévention secondaire. Une enquête familiale approfondie est fondamentale dans tous les cas. L'hypercholestérolémie pure (type IIa) et l'hyperlipidémie combinée (type IIb) sont transmises selon un mode autosomique dominant, et un contrôle biologique des ascendants, des collatéraux et des descendants du propositus est indispensable. Si la physiopathologie de l'hyperlipidémie combinée (type IIb) reste incertaine (elle semble liée à une augmentation de la synthèse hépatique de l'apoprotéine B-100 sans plus de précision sur son mécanisme), celle de l'hypercholestérolémie pure (type IIa) est beaucoup mieux connue. Il est classique aujourd'hui de parler d'hypercholestérolémie familiale et d'hypercholestérolémie polygénique. C'est dans le premier cas que les avancées génétiques ont été considérables avec, soit la mutation du gène $\mathrm{du}$ récepteur des LDL (où les anomalies sont classées en 5 chapitres et où plus de 300 mutations sont actuellement décrites), soit la mutation du $3500^{\mathrm{e}}$ acide aminé de l'apolipoprotéine B-100 (et deux autres mutations plus rares récemment décrites). Les formes hétérozygotes correspondant à ces deux types d'anomalies donnent des tableaux cliniques et biologiques assez comparables, avec peut-être une cholestérolémie plus modérée et des lésions coronaires retardées de 5 à 10 ans dans le syndrome de déficience familiale en apo B-100 [6]. Les formes homozygotes de déficience en apo B100 s'accompagnent d'une hypercholestérolémie relativement modérée [7], contrairement à la forme homozygote du déficit en récepteur LDL [8]. Dans cette affection, si, par définition, un sujet normal a $100 \%$ de récepteurs des LDL au niveau des membranes des cellules hépatiques et de la plupart des autres cellules périphériques extra-hépatiques, un sujet hétérozygote a $50 \%$ de récepteurs, et un sujet homozygote en est dépourvu. On divise cependant les formes homozygotes en receptor negative où il y a moins de $2 \%$ de récepteurs et receptor defective où il y a entre $2 \%$ et $30 \%$ de récepteurs [9]. C'est dans les formes receptor negative que l'on observe les concentrations les plus élevées de CT, entre 6 et $12 \mathrm{~g} / 1$, des complications coronaires très précoces et une espérance de vie qui ne dépasse pas l'âge de 15 ans en l'absence de traitement. Le seul traitement efficace est représenté par les LDL-aphérèses, en attendant dans un avenir plus ou moins proche la thérapie génique dont l'efficacité exigera que beaucoup de problèmes techniques soient résolus [10]. Les statines, dont le rôle est d'augmenter, par rétrocontrôle, le nombre des récepteurs des LDL au niveau des membranes cellulaires, sont efficaces dans les formes hétérozygotes et homozygotes receptor defective, mais inefficaces dans les formes receptor negative. Quelques transplantations hépatiques dans ces formes dramatiques se sont révélées assez efficaces, $70 \%$ des récepteurs des LDL de tout l'organisme étant situés au niveau de la membrane des hépatocytes. La prescription ultérieure de statines peut alors apporter un bénéfice supplémentaire.

Dans les hypercholestérolémies polygéniques, la transmission génétique n'est pas très claire. Il pourrait s'agir d'une association de plusieurs anomalies génétiques (défaut mineur du récepteur LDL, de l'apo B-100 ...). Le phénotype de l'apolipoprotéine E intervient également (l'allèle $\varepsilon 2$ est associé à un concentration d'apo B $10 \%$ plus bas que la moyenne de la population, alors que l'allèle $\varepsilon 4$ est associé à un concentration d'apo B $5 \%$ plus élevé). Cette hétérogénéité du phénotype de l'apo E module la réponse à certains médicaments, le degré d'absorption du cholestérol alimentaire, la réponse au régime « anticholestérol ». C'est un exemple de l'intrication entre facteur(s) génétique(s) et rôle de l'environnement.

\section{En conclusion}

Les travaux fondamentaux de Brown et Goldstein ont fait faire un bond considérable à la connaissance des troubles du métabolisme des lipides. Aujourd'hui, même s'il existe encore quelques points obscurs, la composition des lipoprotéines est parfaitement déterminée, le rôle des différentes apolipoprotéines est bien précisé. Les gènes codant pour les récepteurs, les différentes enzymes (lipoprotéine-lipase, lipase hépatique, lécithine-cholestérol-acyl-transférase), la protéine de transfert (cholesterol ester transfer protein), toutes les apolipoprotéines majeures sont localisés et séquencés. La synthèse intracellulaire du cholestérol est totalement connue. Si bien que le clinicien dispose aujourd'hui d'outils irremplaçables pour le diagnostic des hyperlipidémies génétiquement transmises et pour le traitement de ces maladies métaboliques (le point d'impact et le mécanisme d'action des principaux hypolipidémiants sont précisés pour la plupart)

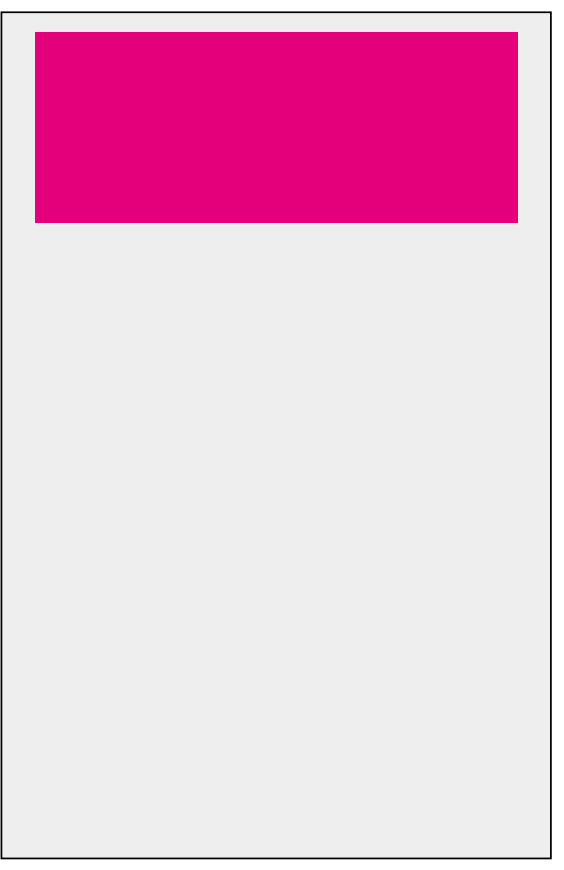

